\title{
同步抽运飞秒光参量振荡器中的载波-包络相位控制 及其应用
}

孙敬华 ${ }^{(2)}$, B. J. S. Gale ${ }^{\circledR}$, D. T. Reid ${ }^{\circledR}$

(1) Ultrafast Optics Group, School of Engineering and Physical Sciences, Heriot-Watt University, Edinburgh EH14 4AS, UK;

(2) 中国科学院物理研究所光物理开放实验室, 北京 100080

E-mail: j.sun@hw.ac.uk

2007-09-01 收稿, 2007-12-11 接受

英国工程与物理科学研究委员会(UK, EPSRC)、美国相干公司(Coherent Inc.)和中国国家自然科学基金(批准号: 60308001)资助项目

摘要 同步抽运飞秒光参量振荡器 $(\mathrm{OPO})$ 以及抽运源所构成的飞秒脉冲系统具有内在同步、多 波长脉冲输出以及各脉冲的载波-包络相位(CEP)的内在关联等特性, 在超宽频率梳以及脉冲 的相干合成方面具有先天的优势. 本文阐述了一系列该研究方向的最新成果, 诸如探讨了 OPO 内载波-包络相位与能量守恒的关系, 并通过实验进行了检验; 利用自参考法控制了作为 抽运源的飞秒钛宝石激光器 CEP, 并通过压电陶瓷(PZT)锁定了其脉冲重复频率; 利用 OPO 产 生的非相位匹配可见光, 并以抽运光的超连续作为参考, 通过 OPO 端镜上的快速 PZT 控制了 OPO 产生的所有脉冲的 CEP, 从而获得 $400 \mathrm{~nm} 2.4 \mu \mathrm{m}$ 超宽频率梳, 控制精度高于 $1.2 \mathrm{kHz}$ 带 宽; 并利用波长相近相位锁定的抽运光以及信号光倍频实现了不同脉冲的相位相干, 为产生 极窄的合成光脉冲打下了基础.

超短光脉冲的时间宽度在达到了皮秒 $(\mathrm{ps})$ 和飞秒 (fs) 以后, 正迈入亚飞秒以及阿秒(as)的量级. 作为最 典型的飞秒脉冲产生装置, 克尔透镜锁模(KLM)钛宝 石激光器可以直接产生包络宽度小于两个振荡周期 的脉冲序列 ${ }^{[11}$. 而放大过的光脉冲经过中空光纤光谱 展宽以后可以压缩到 $2.6 \mathrm{fs}^{[2]}$, 这也是目前在近红外 至可见光范围内得到的最短的脉冲. 当脉冲宽度只 有几个振荡周期以后，脉冲的载波和包络的相对相 位(carrier-envelope phase, CEP)变得越来越重要, 并 成为产生单周期脉冲以至阿秒脉冲, 以及影响超短 脉冲应用等方面的关键因素. 目前人们已经在各种 实验中得到了 CEP可控的超短脉冲, 其产生装置涵盖 从高重复频率的振荡器 ${ }^{[3-6]}$ 到低重复频率但有着高脉

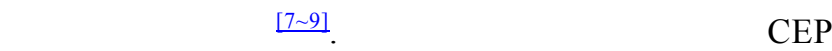
锁定的高功率激光脉冲与稀有气体等物质相互作用 可以产生极紫外(XUV)或软 X射线波段的相干高次 谐波, 通过适当的过滤和压缩, 可以得到一两百阿秒
量级的XUV或软X脉冲 ${ }^{[10 \sim 12]}$ ，使得直接观测原子核 外电子的动力学过程成为可能. 在超國值电离 (above-threshold ionization)方面, 超短脉冲的 CEP, 也就是载波峰值在脉冲包络中的位置, 直接影响几 个周期的超强脉冲同物质相互作用的结果 ${ }^{[13]}$.

根据傅里叶变换原理, 为了在时间域上得到单 振荡周期(可见光范围内)甚至亚飞秒的极窄脉冲包 络，必须具备有极宽的、远大于单个激光增益介质发 射带宽的相干频率成分. 虽然高次谐波产生(HHG)以 及高阶受激拉曼散射过程 ${ }^{[14]}$ 可以实现这一点, 但需 要有很高的脉冲能量. 在低脉冲能量领域, 脉冲的相 干合成提供了一个更为直接的解决方案 ${ }^{[15]}$. 将两个 或多个不同波长的光脉冲序列的重复频率和 CEP 相 互锁定 ${ }^{[16-187}$, 得到具有相干性的、范围极宽的组合光 谱, 从而获得比每个组成脉冲更短的脉冲宽度.

对超短激光脉冲的 CEP进行精确控制还会为光 计量领域带来革命性的影响 ${ }^{[19-21]}$. 锁模激光脉冲的 
光谱为一系列的梳状频率线 (频率梳), 任意一个频率 线的绝对频率可以表示为

$$
f=f_{\text {cep }}+n f_{\text {rep }},
$$

其中 $n$ 为很大的正整数, $f_{\text {rep }}$ 为激光器的重复频率, $f_{\text {cep }}$ 为脉冲CEP的变化频率, 其值在 0 到 $f_{\text {rep }}$ 之间, 是 频率 $f$ 相对于重复频率 $f_{\text {rep }}$ 的 $n$ 阶谐波的偏移量, 也 是整个频率梳相对于一个起点于零点的、同样间隔的 频率梳的偏移量. $f_{\text {rep }}$ 和 $f_{\text {cep }}$ 均在微波频段, 从而(1) 式将光学频率标准和原子钟频率标准直接联系起来. 通过非线性光学作用可以将这一频率梳扩展到从极 紫外到中红外的范围, 使得光学频率的测量变得异 常简单以及具有更高的精度

因此, 如何在各种装置中利用各种手段得到各种 不同波长的 CEP高精度可控的光脉冲成为近几年的研 究热点. 然而大多数的研究都集中于固体激光脉冲方 面, 只有少数涉及到光参量作用产生的光脉冲. 相对于 固体锁模激光器而言, 光参量振荡器 $(\mathrm{OPO})$ 和光参量互 作用在脉冲相干合成以及宽带光频率梳方面具有独特 的优势. 例如, 若利用两个独立的锁模激光器产生具有 相干合成的脉冲序列, 它们必须非常严格地同步 ${ }^{[18.24]}$. 但是作为同步抽运的 $\mathrm{OPO}$, 其产生的各波长的脉冲序 列理论上就是严格地与抽运光同步的, 从而大大简化 了相位控制系统. 此外, 飞秒OPO可以同时产生很多不 同波长的光脉冲. 除了在非线性晶体中抽运光(p)在相 位匹配条件下所产生的信号光(s)和空闲光(i)外, 还有 诸如抽运光和信号光、抽运光和空闲光的和频, 抽运 光、信号光和空闲光的倍频等等非相位匹配脉冲输出. 其脉冲频率涵盖从紫光到中红外的极宽的范围, 为光 频标以及超短脉冲相干合成提供了丰富的光源. 还有 另外一个优点就是在参量互作用中, 各脉冲的相位是 相互耦合在一起的, 这使得控制各脉冲的CEP变化频 率异常简单. 例如在文献[7]中, 研究人员利用光参量 互作用中的抽运光和信号光的差频产生了 $\mathrm{CEP}$ 自稳定 的空闲光脉冲. 以上这些特点使得飞秒 $O P O$ 具有丰富 的研究内容. 本文将讨论飞秒KLM钛宝石激光器及其 同步抽运的飞秒OPO所产生光脉冲的重复频率以及 CEP的控制, 并讨论所产生的多波长相位受控脉冲在 不同方面的应用.

\section{1 实验装置}

图 1中, 飞秒OPO的抽运源是一个自建的飞秒
KLM钛宝石激光器, 输出脉冲的重复频率为 200 $\mathrm{MHz}$, 脉冲半高全宽度 $(\mathrm{FWHM}$ ) 为 $50 \mathrm{fs}$, 光谱宽度 20 $\mathrm{nm}$, 中心波长 $800 \mathrm{~nm}$, 在 $6 \mathrm{~W}$ 绿光(Verdi X)抽运下通 过 $25 \%$ 输出耦合镜 $(\mathrm{OC})$ 产生大于 $1.3 \mathrm{~W}$ 的平均功率. 钛宝石腔内的负色散由一个GTI(Gires-Tournois interferometer)镜片提供. 激光腔的一个端镜安装于 压电陶瓷(PZT1)上用以控制腔长, 并可以通过锁相 环路将其输出脉冲的重复频率锁定在一个稳定的 200 $\mathrm{MHz}$ 参考频率上. 在钛宝石的抽运绿光中插入一个 声光调制器 (AOM)调制抽运光的强度, 影响激光腔 内的非线性折射率, 从而控制输出脉冲序列的 CEP变 化频率. $20 \%$ 的钛宝石脉冲能量被导入到非线性干涉 仪内一段 $30 \mathrm{~cm}$ 长的光子晶体光纤 (PCF)中, 为 $f$-to- $2 f$ 自参考过程产生一个倍频程的超连续 ${ }^{[25]}$. 自参考过 程给出钛宝石激光器的 CEP变化频率 $f_{\mathrm{cep}}^{\mathrm{p}}$, 其拍频信 号由一个硅雪崩二极管(APD1)探测. 剩余的大于 1 $\mathrm{W}$ 的钛宝石光用来抽运以 $\mathrm{MgO}: \mathrm{PPLN}$ 晶体为非线性 介质的OPO, 并在近红外波段产生 $50 \sim 100 \mathrm{~mW}$ 的信 号光输出. 非线性晶体长度 $1 \mathrm{~mm}$, 两面镀 $800 \mathrm{~nm}$ 以 及 1.2 1.6 $\mu \mathrm{m}$ 的增透膜. OPO的腔内色散也是使用 GTI镜片来进行补偿. 图 1 右侧是除了得到 $f_{\mathrm{cep}}^{\mathrm{p}}$ 的非 线性干涉仪以外的第二个干涉仪, 其中两个独立的 延迟线将 $\mathrm{OPO}$ 非相位匹配产生的信号光的倍频 $(2 \mathrm{~s})$ 以及空闲光和抽运光的和频 $(p+i)$ (分别在红光和黄光 波段) 分别与剩余的抽运光超连续产生拍频,

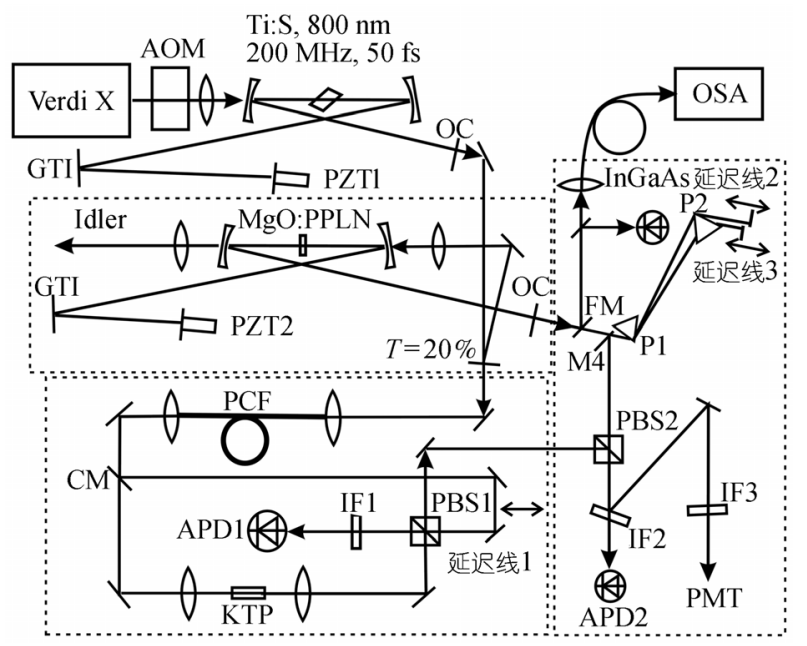

图 1 同步抽运飞秒 $O P O$ 的 CEP 控制实验光路示意图 (部分光学元件未标出)

$\mathrm{CM}$ 为短波反射长波透射镜片; PBS 为偏振分束器; IF 为干涉滤波 片; FM 为可翻转镜片; P 为棱镜; OSA 为光学光谱分析仪 
其拍频信号分别由一个雪崩二极管(APD2)和一个光 电倍增管(PMT)探测, 从而获得 $\mathrm{OPO}$ 所产生的光脉冲 的CEP信息. OPO的一个端镜安装在另一个快速压电 陶瓷(PZT2)上, 用以通过腔长调节控制 $\mathrm{OPO}$ 产生脉 冲的 $\mathrm{CEP}{ }^{[26]}$. OPO的腔长匹配由精密移动平台和另一 块移动范围较大的PZT来实现(图中未标出).

\section{2 参量互作用中的相位关系以及飞秒 OPO 中能量守恒的表达}

在二阶非线性三波互作用中的相位关系可以表 示为 ${ }^{[27]}$

$$
\phi_{\mathrm{p}}=\phi_{\mathrm{s}}+\phi_{\mathrm{i}}-\pi / 2,
$$

其中 $\mathrm{p}, \mathrm{s}$ 和 $i$ 分别对应抽运光、信号光和空闲光. 尽管 直接测量脉冲的相位已经得以实现, 但是要求脉冲 具有极高的峰值功率 ${ }^{228]}$. 在低功率领域, 研究OPO脉 冲的CEP变化频率的关系可以间接地验证关系式(2). 将(2)式两边对时间求导, 就得到载波相位相对于脉 冲包络的变化率的相互关系:

$$
f_{\text {cep }}^{\mathrm{p}}=f_{\text {cep }}^{\mathrm{s}}+f_{\text {cep }}^{\mathrm{i}} \text {. }
$$

式中各项就是在频率域中各脉冲的频率梳相对于起 始于原点的频率梳的偏移 (offset), 它们都小于频率 梳的间隔 $f_{\text {rep }}$. (3)式为(2)式在时间上的变化关系. 即

此外, 理论上参量互作用中的能量是守恒的 ${ }^{[27]}$,

$$
\omega_{\mathrm{p}}=\omega_{\mathrm{s}}+\omega_{\mathrm{i}} .
$$

也就是 $f_{\mathrm{p}}=f_{\mathrm{s}}+f_{\mathrm{i}}$, 式中各项分别为 3 个光脉冲的绝 对频率. 而根据(1)式，各光脉冲的绝对频率可以表 达为

$$
\begin{aligned}
& f_{\mathrm{p}}=j \cdot f_{\text {rep }}+f_{\text {cep }}^{\mathrm{p}}, \\
& f_{\mathrm{s}}=k \cdot f_{\text {rep }}+f_{\text {cep }}^{\mathrm{s}}, \\
& f_{\mathrm{i}}=l \cdot f_{\text {rep }}+f_{\text {cep }}^{\mathrm{i}},
\end{aligned}
$$

式中 $j, k, l$ 均为整数. 将(5)式代入(4)式, 消去 $f_{\text {rep }}$, 则得到(3)式. 也就是说, (3)式也可以用来检验在光参 量过程中的能量守恒关系.

参量互作用过程, 尤其是在飞秒脉冲范围内, 由 于极高的脉冲峰值功率, 很容易会伴随其他非线性 现象发生，比如拉曼散射、布里渊散射、自相位调制、 四波混频等 ${ }^{[29,30]}$. 这些非线性现象都会破坏参量过 程的能量守恒关系. 在文献[31,32]中, 人们在连续光 倍频以及 $\mathrm{OPO}$ 中检验了二阶非线性现象的频率分配
(能量分配)精度, 并在实验上分别达到了 $10^{-13}$ 和 $10^{-18}$ 量级, 但是他们都是将实验设定在特定的波长. 例如在文献[32]中, OPO是用 $\mathrm{Nd}$ : $\mathrm{YAG}$ 的倍频来抽运, 并且工作于简并点附近, 从而使得抽运光的基频、信 号光和空闲光的频率都特别接近, 这样它们之间的拍 频信号都在微波领域, 以利于精确的频率比较. 而在 飞秒领域，如上面讨论的，参量过程的能量守恒关系 可以用(3)式来取代, 其最大的优势在于(3)式中所有 的这些频率都在微波频段, 不论OPO工作于哪个波 长.

在图 1 的第二个干涉仪中, APD2 探测到的信号光 倍频同抽运光超连续的拍频信号为 $f_{\text {beat }}^{\text {red }}=2 f_{\text {cep }}^{\mathrm{s}}-f_{\text {cep }}^{\text {p }}$, PMT 探测到的空闲光和抽运光和频与抽运光超连续 之间产生的拍频信号直接为空闲光的 CEP 变化频率: $f_{\text {beat }}^{\text {yellow }}=\left(f_{\text {cep }}^{\mathrm{i}}+f_{\text {cep }}^{\mathrm{p}}\right)-f_{\text {cep }}^{\mathrm{p}}=f_{\text {cep }}^{\mathrm{i}}$. 根据(3)式可以得到 在图 1 中 3 个探测器所探测的信号满足以下关系:

$$
f_{\text {cep }}^{\mathrm{p}}=f_{\text {beat }}^{\text {red }}+2 f_{\text {beat }}^{\text {yellow }},
$$

(6)式是(3)式在实验中的表现形式. 在 OPO 中, 如果 有伴随的非线性现象发生，使得三波互作用的同时 还有其他的声子或光子产生, 那么由(3)式表达的能 量守恒定律就不成立, 而且在(6)式中就会有一个频 率差值 $\Delta f$ 产生:

$$
\Delta f=f_{\text {cep }}^{\mathrm{p}}-f_{\text {beat }}^{\text {red }}-2 f_{\text {beat }}^{\text {yellow }} .
$$

在实验中, $\mathrm{OPO}$ 是自由工作状态, 也就是没有任 何的相位以及重复频率的锁定. 此时钛宝石的 $f_{\mathrm{cep}}^{\mathrm{p}}$ 的 抖动范围小于 $200 \mathrm{kHz}(5 \mathrm{~s}$ 之内), 而 $\mathrm{OPO}$ 的光脉冲和 抽运光超连续的拍频信号的抖动范围则在 $10 \mathrm{MHz}$ 范 围. 为了减小实验误差, 实验中 3 个探测器的信号同 时由同一台频谱分析仪记录. 这样在一个扫描周期 内 $(5 \mathrm{~ms}), 3$ 个信号之间的相对抖动被控制在 $2 \mathrm{MHz}$ 以内. 实验中记录了 25 个数据点, $f_{\text {cep }}^{\mathrm{p}}, f_{\text {beat }}^{\text {red }}$ 和 $f_{\text {beat }}^{\text {yellow }}$ 分别在 $64 \mathrm{MHz}, 33 \mathrm{MHz}$ 和 $48 \mathrm{MHz}$ 附近. 统计

$$
\Delta f=2 f_{\text {beat }}^{\text {yellow }}-f_{\text {beat }}^{\text {red }}-f_{\text {cep }}^{\text {p }}
$$

的结果得到 $\Delta f$ 平均值为 $50 \mathrm{kHz}$ (远小于一般非线性 现象所产生的声子或光子的频率), 标准偏差 1.78 MHz. (8)式和(7)式的差别在于符号不同, 这是因为 在实际探测中, 我们将实验数据局限于 $0 \sim 100 \mathrm{MHz}$ 的范围内, 所以探测到的拍频信号是实际频率的绝 对值, 或者是从 $200 \mathrm{MHz}$ 重复频率处产生的边带谐 波. 但这从理论上不影响实验检测(7)式中的 $\Delta f$ 值. 
统计结果说明, 在实验误差范围内该 $\mathrm{OPO}$ 中能量是 守恒的. 此外在文献[33]中我们通过记录另外一组数 据也得到了类似的实验结果. 该实验的最大优势在 于不需要任何控制措施，并且 $\mathrm{OPO}$ 可以工作于任何 可以达到的波长. 如果锁定 $\mathrm{OPO}$ 的 CEP变化频率, 例 如我们在下面要讨论的实验中将 $\mathrm{OPO}$ 的 $\mathrm{CEP}$ 变化频 率锁定在了 $1.2 \mathrm{kHz}$ 的带宽范围内 ${ }^{[34]}$, 那么实验结果 将更加精确.

检测参量过程中的能量守恒在很多领域具有现 实意义, 例如在基于参量下转换过程的量子信息实 验中 ${ }^{[35]}$, 如果能量守恒被破坏, 那么所产生的光子不 再具有反相关特性, 测量一个纠缠态的光子状态将 不再能够获得另一个光子的全部信息.

\section{3 飞秒 OPO 的 CEP 控制以及超宽带光频 率梳的产生}

如前所述, 飞秒 OPO 在宽带光频率梳的产生方 面具有先天的优势, 那就是所有的光脉冲与抽运源 都是内在同步的，也就是所有的光频率梳具有一致
的频率间隔. 那么只要控制抽运源的重复频率以及 所有光脉冲的 $\mathrm{CEP}$ 变化频率, 我们就可以在从紫光到 中红外的超宽频谱范围内得到稳定的频率梳 ${ }^{[34]}$.

在本实验中, 信号光和空闲光分别在 1.3 和 2.08 $\mu \mathrm{m}$ 附近. 非相位匹配的抽运光、信号光和空闲光的 倍频分别为 $400 \mathrm{~nm}(2 \mathrm{p}), 650 \mathrm{~nm}$ (2s, 红色)和 $1.05 \mu \mathrm{m}$ (2i), 信号光与抽运光、空闲光与抽运光的和频分别 在 $480 \mathrm{~nm}(\mathrm{p}+\mathrm{s})$ 和 $580 \mathrm{~nm}(\mathrm{p}+\mathrm{i}$, 黄色)附近(见图 2(a)). 非相位匹配的这些光脉冲的平均功率都大于 $2 \mathrm{~mW}$, 而且几乎从每一个腔镜都可以透射出一部分来, 十 分有利于探测和利用.

图 3 为实验系统的CEP控制示意图. 钛宝石的重 复频率通过其端镜上的PZT1 被锁定在一个外部 200 MHz的时钟(PRL-175NT-200)上，因而所有的、包括 OPO 产生的所有脉冲的重复频率都被锁定在外部时 钟上. 在CEP变化频率的锁定方面, 由于其很宽的抖 动范围 $(\mathrm{MHz})$, 几乎不可能用一个普通的差频方法来 产生反馈控制信号, 所以利用一个相位频率探测器 $(\mathrm{PFD})^{[36]}$ 将 $\mathrm{CEP}$ 的变化频率同外部参考源的差值转

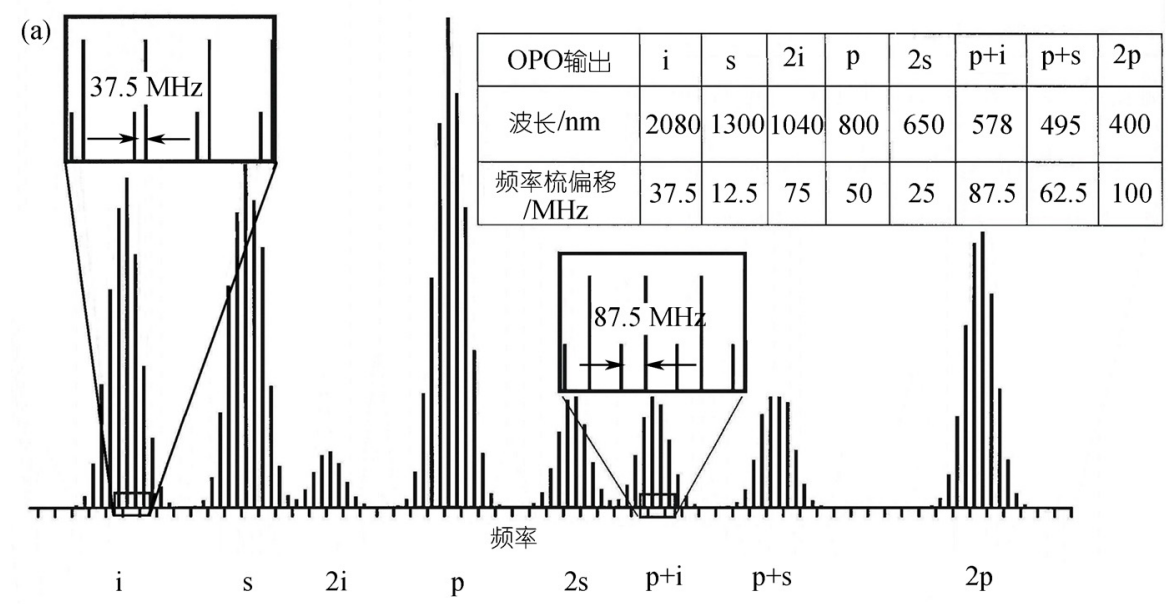

(b)

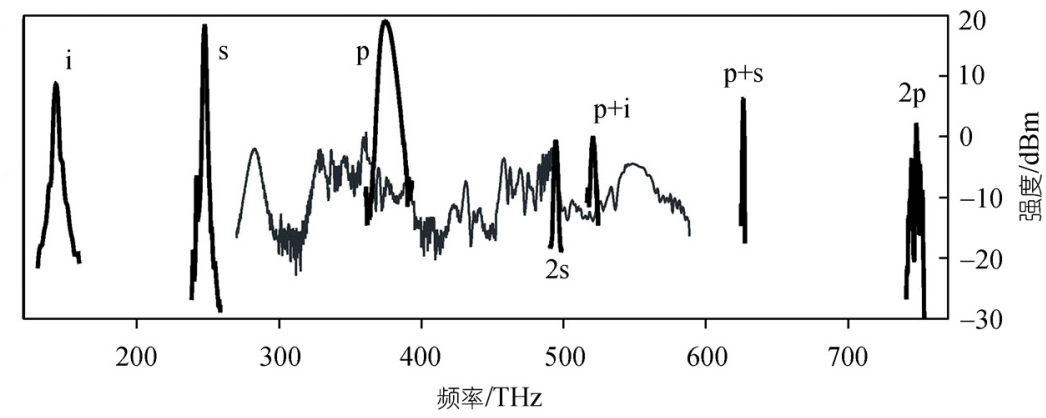

图 2 宽带组合频率梳示意图(a)以及实验获得的稳定的频率成分(b)

(a)中插入的表格给出了 $\mathrm{OPO}$ 的一种可能的波长组合以及一种可能的频率梳偏移量组合; (b)中的灰线为频率稳定的抽运光超连续 
换成频率稍低反馈控制电压信号. 根据(6)式，只要 控制 $f_{\text {cep }}^{\mathrm{p}}$, 然后再控制 $f_{\text {beat }}^{\text {red }}$ 和 $f_{\text {beat }}^{\text {yellow }}$ 中的任意一个, 就可以控制 OPO 产生的所有光脉冲的 CEP 变化频率, 包括非相位匹配的脉冲. 在本实验中, 我们将 APD1 探测到的 $f_{\text {cep }}^{\mathrm{p}}$, 通过 PFD1 和锁相环控制 AOM, 锁定 在 $50 \mathrm{MHz}$; 将 APD2 探测到的 $f_{\text {beat }}^{\text {red }}$, 通过 PFD2 控制 OPO 端镜上的 PZT2, 锁定在 $25 \mathrm{MHz}$. 在 PFD2 的输 出端口检测到误差信号已经从未锁定时的类矩形波 变成了只有轻微波动的较稳定的信号, 标志着较为 稳定的相位锁定 (见图 4(a)). 光电倍增管探测得到的 信号 $f_{\text {cep }}^{\mathrm{i}}$ 可以用来作为锁相环外部的独立的检验信 号以判定频率锁定的精度. 实验中 PMT 所探测到的 频率为 $37.5 \mathrm{MHz}$ ，在 $-3 \mathrm{~dB}$ 处的带宽约为 $1.2 \mathrm{kHz}$ (见 图 4(b)), 接近频谱分析仪 $1 \mathrm{kHz}$ 的分辨率(Agilent

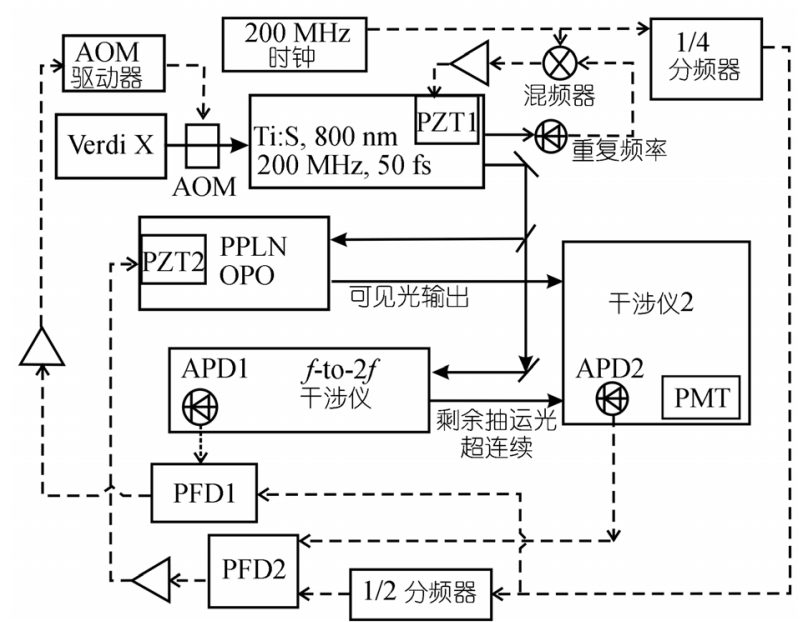

图 3 同步抽运飞秒 OPO 中 CEP 锁定反馈系统示意图
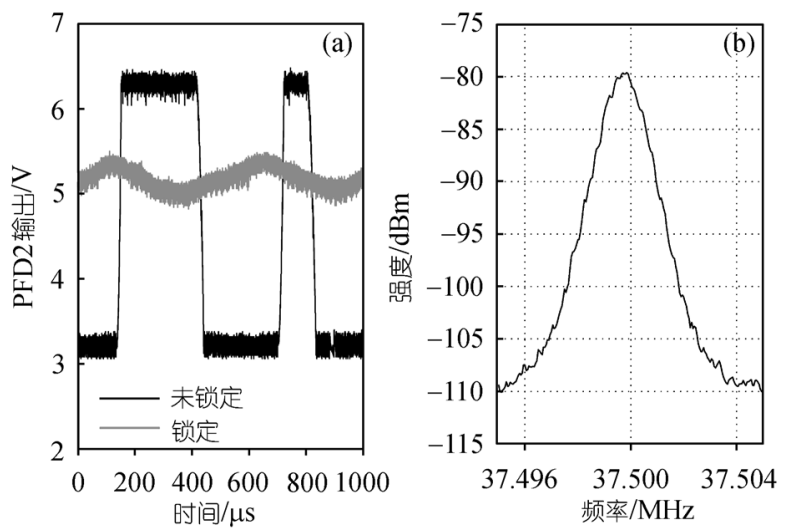

图 4 相位频率探测器输出端的误差信号(a), 以及相位锁 定后 PMT 上探测到的拍频信号(b)
E4411B). 所以实际的数值可能更窄. 3 个探测器所得 的频率满足关系式(6). 值得注意的是, 图 2 中用来产 生参考频率的两个分频器的分频倍数不必是固定的, 甚至可以用任意稳定的参考频率来取代它们. 所以 原则上所有这些频率梳的绝对位置以及相对位置可 以被锁定在任意参考频率上, 这为它们的应用提供 了更大的灵活性. 考虑到该OPO的调谐能力, 所有锁 定的频率梳可以跨越从 $400 \mathrm{~nm}$ 到 $2.4 \mu \mathrm{m}$ 的大于 2.5 个倍频程的广大范围. 而且这还不是这类OPO所能 产生的光谱范围的极限. 在红外波段, 基于PPLN的 OPO的空闲光频率可以达到 $6.8 \mu \mathrm{m}^{[37]}$. 这种组合的 频率梳的缺陷是, 由于每一个脉冲各自的带宽有限, 它们的光谱并不能互相衔接(见图 2(b)), 所以合成的 大于 2.5 个倍频程的频率梳是间断的, 但是通过 $\mathrm{OPO}$ 的调谐仍然能够使其产生的频率梳有效覆盖其范围 内几乎所有的频率.

\section{4 利用飞秒 OPO 产生的脉冲进行脉冲相干 合成}

在上一节中为了得到稳定的频率梳需要锁定脉 冲的重复频率, 但是在相干合成方面则无此必要, 只 需要同步即可. 而从同步抽运飞秒OPO及其抽运源 中输出的所有的脉冲都是同步的, 这为超短脉冲, 甚 至单周期脉冲的相干合成提供了极大的便利. 在文 献 [38]中 Kobayashi等人正是利用OPO的这一特点, 并且让其工作于特定的频率, 即抽运光、信号光和空 闲光的频率之比为 $3: 2: 1$, 实现了不同波长脉冲的相 位相干. 假设在该OPO中抽运光的频率为 $3 \omega$, 则信 号光和空闲光的频率分别为 $2 \omega$ 和 $\omega$. 在开始时刻, 三 脉冲的载波峰值均在包络的中央, 可以在包络的中 央产生干涉加强. 在下一个脉冲组到来的时刻, 要想 三个脉冲仍旧能够产生干涉加强, 则在时间上各载 波的峰值相对于包络的偏移量应该是相同的, 但是 由于各脉冲的频率成 $3: 2: 1$ 的关系, 所以它们相位的 偏移量之比 $\Delta \phi_{\mathrm{p}}: \Delta \phi_{\mathrm{s}}: \Delta \phi_{\mathrm{i}}$ 也应该是 $3: 2: 1$ 的关系. 由 于重复频率 $f_{\text {rep }}$ 都相同, 根据 $f_{\text {cep }}=f_{\text {rep }} \Delta \phi / 2 \pi$, 可得 $f_{\text {cep }}^{\mathrm{p}}: f_{\text {cep }}^{\mathrm{s}}: f_{\text {cep }}^{\mathrm{i}}=3: 2: 1$. 而且由于上述频率比例关系, 抽运光和空闲光的和频以及信号光的倍频均为 $4 \omega$, 它们之间可产生拍频信号 $2 f_{\mathrm{cep}}^{\mathrm{p}}-3 f_{\mathrm{cep}}^{\mathrm{s}}$. 只需将这一 拍频信号锁定在 0 值或者 $f_{\text {rep }}$ 的若干分之一, 均可以 得到相位相干的脉冲合成. 
在文献[39]中我们提出了另一个更为简便的利 用双色飞秒 $\mathrm{OPO}^{[40]}$ 产生合成脉冲的方案. 在该双色 OPO腔内由GTI镜所提供的负色散和材料以及镜片 的正色散相结合后, 使得信号光脉冲在 $1.26 \mu \mathrm{m}$ 附近 时在腔内的群速色散接近零值, 在短于 $1.26 \mu \mathrm{m}$ 时为 正值, 而在大于 $1.26 \mu \mathrm{m}$ 时为负值. 通过适当的腔长 调谐, 可以使得在这两个色散区各有某一波长的脉 冲具有相同的时间延迟, 从而都可以在腔内振荡. 有 趣的是, 这两个不同波长的信号光脉冲具有不同的 CEP变化频率 $f_{1}$ 和 $f_{2}$ (分别对应短波长和长波长), 而 且由于它们本身就是同步的，所以，尽管它们之间只 有极其微弱的频率重叠都可以产生一个等于其 CEP 变化频率之差的拍频信号. 我们称之为内部拍频

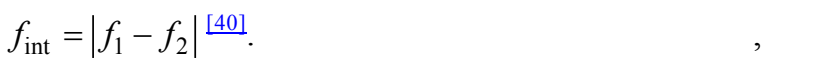
个很自然的想法就是利用这个内部产生的频率差来 “纠正” 其中的一个信号光脉冲, 使它与另一个信号 光脉冲具有相同的CEP变化频率以形成干涉叠加, 产 生具有更短时间宽度的波形.

图 5 为利用双色 $\mathrm{OPO}$ 来实现脉冲合成的实验示 意图. 实验中 $\mathrm{OPO}$ 输出的双波长信号光通过一个 $\mathrm{AOM}$ 后产生 +1 级衍射, 然后 0 级衍射光被原路返回 以产生-1 级衍射光. 同时由于不同的波长经过 $\mathrm{AOM}$ 后具有不同的空间衍射角, 两个不同波长的信号光 脉冲衍射后空间上也是分开的. 正负衍射光脉冲经 过一个双色镜又合并成 A, B 两束. 若 AOM 的驱动频 率为 $f_{\mathrm{AOM}}$, 那么在 $\mathrm{A}$ 光束中, 短波长脉冲经过- 1 级 衍射后其 CEP 变化频率 (频率梳的偏移量) 为 $f_{1}-f_{\mathrm{AOM}}$, 长波长脉冲经过 +1 级衍射后为 $f_{2}+f_{\mathrm{AOM}}$, 两者的频率差为 $f_{1}-f_{2}-2 f_{\mathrm{AOM}}$. 如果 $f_{1}>f_{2}$, $f_{\mathrm{AOM}}=f_{\text {int }} / 2$, 那么可以得到两个波长的光脉冲在 $\mathrm{A}$ 光束中具有相同的 CEP 变化频率, 两者是相位相干 的. 反之, 如果是 $f_{1}<f_{2}$, 则是在 $\mathrm{B}$ 光束中得到相干

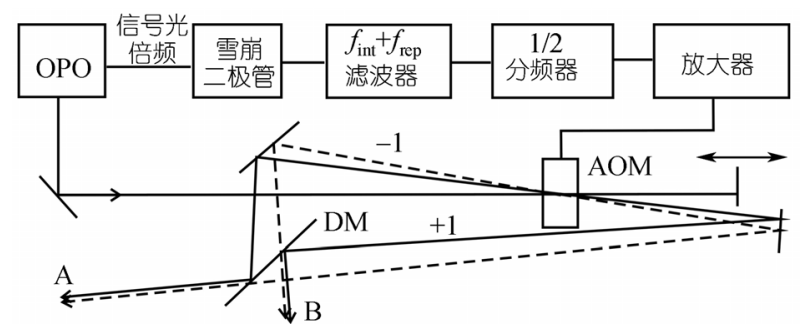

图 5 利用双色 OPO 产生的双波长信号光脉冲进行相干 合成实验的示意图

虚线为长波长脉冲; DM 为双色镜
的两个脉冲. 至于具体是哪个组合是相干的, 可以通 过实验检测出来. 由于实验中采用的 $\mathrm{AOM}$ 的中心频 率在 $111 \mathrm{MHz}$, 所以实际采用 $\left(f_{\text {rep }}+f_{\text {int }}\right) / 2$ 而非 $f_{\text {int }} / 2$ 来驱动 $\mathrm{AOM}$, 其结果是引入了 $2 \pi$ 的相位差, 但不影响相干性.

由于两个脉冲的中心波长相差大于 $100 \mathrm{~nm}$, 而 且在外部两者的波长几乎是不连贯的, 无法用互相 关等方法检测相干性 ${ }^{[16]}$. 为了检验是在 $\mathrm{A}$ 光束还是 在 $\mathrm{B}$ 光束中得到了相干的两个脉冲, 我们用部分钛宝 石脉冲能量抽运一根 $70 \mathrm{~cm}$ 长的光子晶体光纤 $(\mathrm{PCF}$, Crystal Fibre NL-2.0-740), 产生波长可以达到 1.35

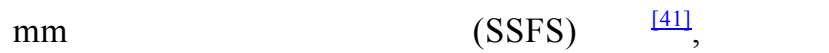
调制后的短波长和长波长的两个信号光脉冲产生拍 频来检测它们的CEP变化频率 ${ }^{[39]}$. 如果它们的拍频 是一致的, 则说明两个信号光脉冲的相位变化相同. 实验结果证明, 在 A光束中两个信号光同 SSFS脉冲 的拍频是相同的, 而在 B中两者则相差 $2 f_{\text {int }}$ (此处为 $55 \mathrm{MHz}$ ). 通过互相关频率分辨光学开关法 (XFROG) 来单独测量 $\mathrm{A}$ 光束中两个信号光脉冲的振幅和相位, 可以推测此处相干合成的波形为一个由若干 $30 \mathrm{fs}$ 宽 度的超短脉冲组成的脉冲序列(见图 6).

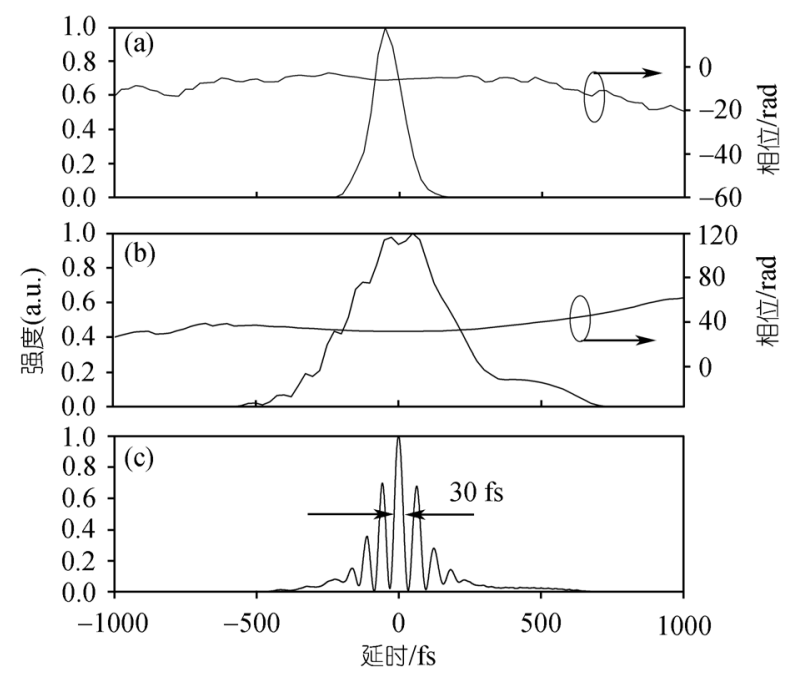

图 6 XFROG 分析结果

(a)和(b)为两个波长的信号光脉冲的时间包络以及相位, (c)为计算 出的相干合成后的脉冲波形

上面两种相干合成方法有一个共同的不足之处 在于合成脉冲的 CEP 仍旧是没有锁定的. 在上一节 的描述中, 我们已经得到了相对位置和绝对位置都 锁定了的组合频率梳. 在此基础上, 通过选择适当的 
频率梳偏移量, 也就是选择适当的 CEP 变化频率，可 以使得不同波长的多个脉冲具有相同的 CEP 变化频 率, 形成相干合成脉冲. 例如, 将 $f_{\mathrm{cep}}^{\mathrm{p}}$ 锁定在 $50 \mathrm{MHz}$, $f_{\text {beat }}^{\text {yellow }}$ (也就是 $f_{\text {cep }}^{\mathrm{i}}$ ) 锁定在 $25 \mathrm{MHz}$, 那么根据(3)式, $f_{\text {cep }}^{\mathrm{s}}$ 也是 $25 \mathrm{MHz}$. 这样抽运光、信号光的倍频以及空 闲光的倍频都具有 $50 \mathrm{MHz}$ 的 CEP 变化频率，也就是 说这 3 个脉冲的相对相位都是固定的，从而可以实现 相干合成. 结合 OPO 的可调谐性, 可以在频率域得 到不同的合成光谱，在时间上获得不同包络的相干 波形. 在实验上, 我们对图 1 中 OPO 及控制系统做了 部分改动(见图 7). OPO 腔内添加了一块 BBO 倍频晶 体, 以获得较强的信号光的倍频信号, 并且更换了镜 片组, 使得信号光的调谐范围在 $1.4 \sim 1.56 \mu \mathrm{m}$. 这样, 信号光倍频、抽运光以及空闲光倍频的光谱十分接近 甚至可以相互重叠, 有利于检测实验结果的相干性. 同锁定频率梳的实验一样, 钛宝石的 CEP 的变化频 率通过非线性 $f$-to- $2 f$ 干涉仪、 PFD1 以及 AOM 等器 件组成的反馈环路锁定在 $50 \mathrm{MHz}\left(1 / 4 f_{\text {rep }}\right)$. OPO 的 GTI 镜置于 PZT2 上. 干涉仪 2 用来获得从 PPLN 晶 体表面反射的可见光 $(\mathrm{p}+\mathrm{s})$ 与剩余的抽运光超连续产 生的拍频 $f_{\text {cep }}^{\mathrm{s}}$. PFD2 等反馈环路通过控制 PZT2 将信 号光的 CEP 变化频率锁定在 $25 \mathrm{MHz}\left(1 / 8 f_{\text {rep }}\right)$. 信号 光波长可在 1400 1560 nm 范围内调谐, 这样其倍频 为 700 780 nm 左右. 通过一个 $10 \mathrm{~nm}$ 带宽的干涉滤 光片 IF 可以从 $O P O$ 剩余的抽运光中提取 780 820 nm 范围内不同的光谱成分用来与信号光倍频产生相干 合成. 当然这个滤光片不是必须的, 在本实验中是为 了能得到不同波长和强度相当的抽运光成分.

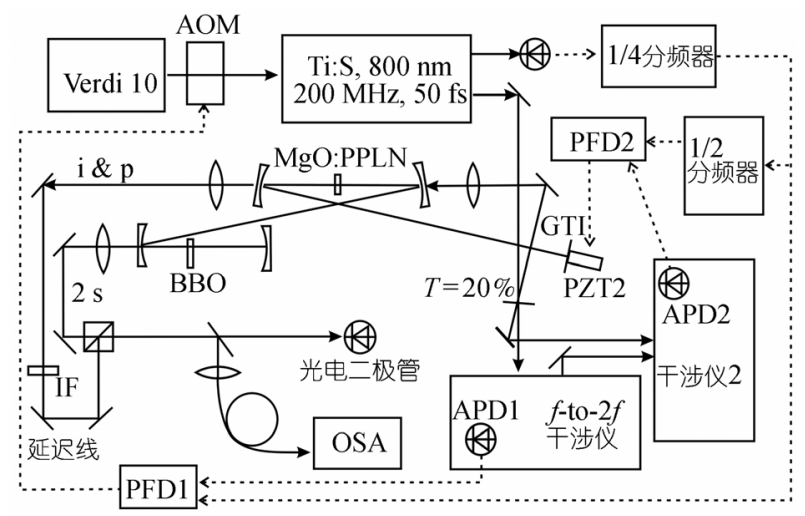

图 7 利用 OPO 的抽运光以及信号光倍频产生相干合成 脉冲的示意图
图 8 显示了当信号光倍频以及滤波后的抽运光 中心波长都在 $780 \mathrm{~nm}$ 附近的光谱干涉实验结果. 当 钛宝石和 OPO 的相位都锁定以后, 调节延迟使两脉 冲重叠, 在光谱上可以看到十分清晰的干涉条纹(图 8(a)). 而在未锁定状态则没有干涉条纹(图 8(b)). 图 $8(\mathrm{c})$ 和(d)为各自单独的光谱.

图 9 为两个脉冲时间上的互相关曲线. 可以看到, 当两个脉冲的 CEP 没有锁定时, 互相关曲线只有一 个强度包络(图 9(a)). 锁定以后, 它们的互相关条纹 有接近于 1:8 比值的包络(图 9(b)), 但是包络内的条 纹不是很整齐, 说明在每一次互相关扫描的时间内 $(20 \mathrm{~ms})$ 两个脉冲的相对相位有抖动. 这一相位噪声 部分来自脉冲互相关前两光路之间的机械振动, 还

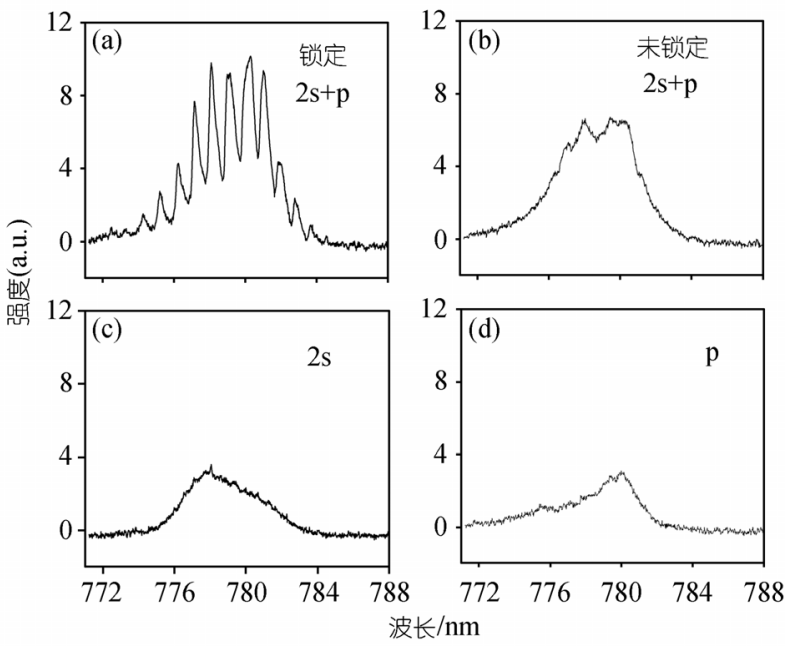

图 8 光谱干涉的测量结果

(a) 相位锁定的信号光倍频光和经过滤光片后的抽运光的叠加; (b) 未锁定的叠加; (c) 只有信号光的倍频; (d) 只有经过滤光片后的 抽运光
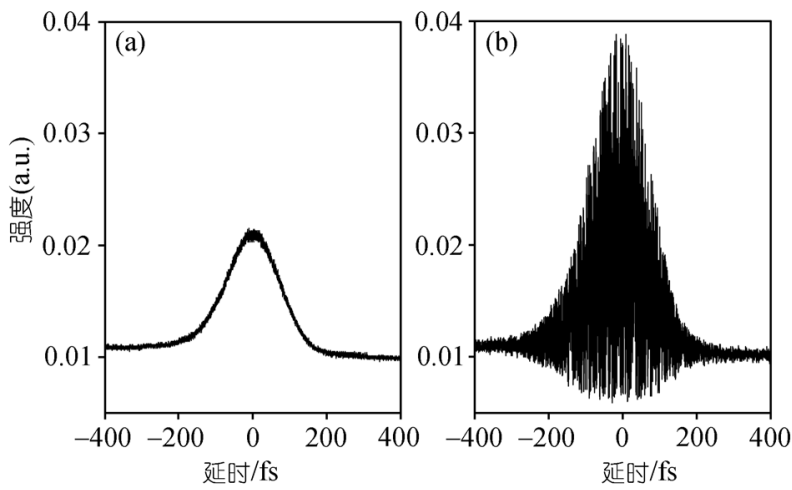

图 9 脉冲互相关曲线

(a) 相位未锁定时信号光倍频与滤波后的抽运光的互相关曲线; (b) 锁定后的互相关曲线 
有就是相位锁定系统本身的比较高频的噪声。为 了得到更为清晰的互相关曲线，下一步的工作是提 高相位频率探测器(PFD)的分辨率, 在 OPO 的相位控 制系统中加入低频率锁相环路 ${ }^{[42]}$, 增加PZT驱动的 带宽, 以提高 OPO 的 CEP 锁定精度, 延长脉冲相干 时间.

\section{5 结论}

本文中我们主要讨论了飞秒同步抽运光参量振 荡器, 包括其抽运源钛宝石 KLM 激光器的脉冲重复 频率以及载波-包络相位控制, 以及这一技术在探讨 光参量过程中的能量守恒、产生超宽带合成频率梳以 及超短脉冲的相干合成等方面的应用. 在实验中我 们成功地将 OPO 的 CEP 变化频率锁定在 $1.2 \mathrm{kHz}$ 的 带宽范围内; 产生了 $400 \mathrm{~nm} 2.4 \mu \mathrm{m}$ 的合成频率梳, 并且其中每个频率梳的绝对位置和相对位置都可以
锁定在任意外部的微波参考频率上 $(0 \sim 200 \mathrm{MHz})$; 通 过将抽运光和信号光的 CEP 变化频率分别锁定在 $1 / 4$ 和 $1 / 8$ 脉冲重复频率上, 得到了抽运光脉冲与信号光 倍频脉冲的相位相干; 以及利用双色 OPO 产生的双 波长信号光脉冲合成了 $30 \mathrm{fs}$ 宽度的脉冲序列. 这些 实验结果说明对于 OPO 的 CEP 控制具有广泛的实际 意义. 但同时, 由于 OPO 的相位由端镜上的 PZT 来 控制, 尽管实验中 OPO 端镜上的 PZT 的谐振频率为 $261 \mathrm{kHz}$ (Thorlabs, AE0203D04F), 但通常所能得到的 驱动器的带宽都偏低, 所以对于频率稍高的噪声的 抑制不够, 这在一定程度上影响最后的锁定带宽. 进 一步的研究工作是提高 OPO 相位控制的精度, 改进 OPO 的光学设计以获得更高的转换效率和更宽的各 组成光谱的宽度, 从而在 $800 \mathrm{~nm}$ 左右很大的范围内 获得长时间相位相干的合成光谱, 通过适当的色散 补偿，实现清晰的超短脉冲时间上压缩的过程.

致谢 感谢中国科学院物理研究所魏志义研究员及其课题组成员的愉快合作.

\section{参考文献}

1 Ell R, Morgner U, Kärtner F X, et al. Generation of 5-fs pulses and octave-spanning spectra directly from a Ti:sapphire laser. Opt Lett, 2001, 26(6): 373-375[DOI]

2 Matsubara E, Yamane K, Sekikawa T, et al. Generation of $2.6 \mathrm{fs}$ optical pulses using induced-phase modulation in a gas-filled hollow fiber. J Opt Soc Am B, 2007, 24(4): 985-989[DOI]

3 Mücke O D, Ell R, Winter A, et al. Self-referenced $200 \mathrm{MHz}$ octave-spanning Ti:sapphire laser with 50 attosecond carrier-envelope phase jitter. Opt Express, 2005, 13(13): 5163-5169 [DO]

4 Kim K, Washburn B R, Wilpers G, et al. Stabilized frequency comb with a self-referenced femtosecond Cr:forsterite laser. Opt Lett, 2005, 30(8): 932-934[DOI]

5 Swann W C, McFerran J J, Coddington I, et al. Fiber-laser frequency combs with subhertz relative linewidths. Opt Lett, 2006, 31(20): 3046 - 3048[DOI]

6 韩海年, 张炜, 王鹏, 等. 飞秒钛宝石光学频率梳的精密锁定. 物理学报, 2007, 56(5): 2760-2764

7 Baltuška A, Fuji T, Kobayashi T. Controlling the carrier-envelope phase of ultrafast light pulses with optical parametric amplifiers. Phy Rew Lett, 2002, 88(13): $133901 \underline{\text { DOI] }}$

8 Gagnon E, Thomann I, Paul A, et al. Long-term carrier-envelope phase stability from a grating-based, chirped pulse amplifier. Opt Lett, 2006, 31(12): 1866-1868 [DOI]

9 Zinkstok R Th, Witte S, Hogervorst W, et al. High-power parametric amplification of 11.8-fs laser pulses with carrier-envelope phase control. Opt Lett, 2005, 30(1): 78-80[DOI]

10 Baltuška A, Udem Th, Uiberacker M, et al. Attosecond control of electronic processes by intense light fields. Nature, 2003, 421: 611$615 \underline{\text { DOI] }}$

11 Sansone G, Benedetti E, Calegari F, et al. Isolated single-cycle attosecond pulses. Science, 2006, 314: 443-446[DOI]

12 Schultze M, Goulielmakis E, Uiberacher M, et al. Powerful 170-attosecond XUV pulses generated with few-cycle laser pulses and broadband multilayer optics. New J Phys, 2007, 9: 243[DOI]

13 Paulus G G, Lindner F, Walther H, et al. Measurement of the phase of few-cycle laser pulses. Phys Rev Lett, 2003, 91(25): $253004 \underline{\text { DOI] }}$

14 Shverdin M Y, Walker D R, Yavuz D D, et al. Generation of a single-cycle optical pulse. Phys Rev Lett, 2005, 94(3): 033904[DOI] 
Hänsch T W. A proposed sub-femtosecond pulse synthesizer using separate phase-locked oscillators. Opt Commun, 1990, 80(1): 71$75 \underline{[\mathrm{DOI}]}$

Shelton R K, Ma L -S, Kapteyn H C, et al. Phase-coherent optical pulse synthesis from separate femtosecond lasers. Science, 2001, 293: 1286-1289[DOI]

Bartels A, Newbury N R, Thomann I, et al. Broadband phase-coherent optical frequency synthesis with actively linked Ti:sapphire and Cr:forsterite lasers. Opt Lett, 2004, 29(4): 403-405[DOI]

Kobayashi Y, Yoshitomi D, Kakehata M, et al. Long-term optical phase locking between femtosecond Ti:sapphire and Cr:forsterite lasers. Opt Lett, 2005, 30(18): 2496-2498[DOI]

Telle H R, Steinmeyer G, Dunlop A E, et al. Carrier-envelope offset phase control: A novel concept for absolute optical frequency measurement and ultrashort pulse generation. Appl Phys B, 1999, 69: 327-332[DOI]

Reichert J, Holzwarth R, Udem Th, et al. Measuring the frequency of light with mode-locked lasers. Opt Commun, 1999, 172: 59-68 $\underline{\text { DOI] }}$

Cundiff S T, Ye J. Colloquium: Femtosecond optical frequency combs. Rev Mod Phys, 2003, 75(1): 325—342[DOI]

Hänsch T W. Nobel lecture: Passion for precision. Rev Mod Phys, 2006, 78(4): 1297-1309 [DOI]

Udem Th, Holzwarth R, Hänsch T W. Optical frequency metrology. Nature, 2002, 416: 233-237[DOI]

Wei Z, Kobayashi Y, Torizuka K. Passively synchronization between femtosecond Ti:sapphire and Cr:forsterite lasers. Appl Phys B, 2002, 74: S171-S176[DOI]

Jones D J, Diddams S A, Ranka J K, et al. Carrier-envelope phase control of femtosecond mode-locked lasers and direct optical frequency synthesis. Science, 2000, 288: 635-639 [DOI]

沈元壤. 非线性光学原理(上册). 北京: 科学出版社, 1987. 125-126

Goulielmakis E, Uiberacker M, Kienberger R, et al. Direct measurement of light waves. Science, 2004, 305: 1267-1269 [DOI]

ond soliton pulse compression. Opt Lett, 2005, 30(4): 364-366[DOI]

Abedin K S. Observation of strong stimulated Brillouin scattering in single-mode As2Se3 chalcogenide fiber. Opt Express, 2005, 13(25): $10266-10271$ [DOI]

Wynands R, Coste O, Rembe C, et al. How accurate is optical second-harmonic generation. Opt Lett 1995, 20(10): 1095-1097

Ikegami T, Slyusarev S, Ohshima S, et al. Accuracy of an optical parametric oscillator as an optical frequency divider. Opt Commun, 1996, 127: 69-72[DOI]

Sun J, Gale B J S, Reid D T. Testing the parametric energy conservation law in a femtosecond optical parametric oscillator. Opt Express, 2007, 15(7): 4378-4384[DOI]

Sun J, Gale B J S, Reid D T. Composite frequency comb spanning 0.4 2.4 mm from a phase-controlled femtosecond Ti:sapphire laser and synchronously pumped optical parametric oscillator. Opt Lett, 2007, 32(11): 1414-1416 [DOI]

Grice W P, Walmsley I A. Spectral information and distinguishability in type-II down-conversion with a broadband pump. Phys Rev A, 1997, 56(2): 1627-1634[DOI]

Prevedelli M, Freegarde T, Hänsch T W. Phase locking of grating-tuned diode lasers. Appl Phys B, 1995, 60: S241—S248

Loza-Alvarez P, Brown C T A, Reid D T, et al. High-repetition-rate ultrashort-pulse optical parametric oscillator continuously tunable from 2.8 to $6.8 \mu \mathrm{m}$. Opt Lett, 1999, 24(21): 1523-1525

Kobayashi Y, Takada H, Kakehata M, et al. Phase-coherent multicolour femtosecond pulse generation. Appl Phys Lett, 2003, 83(5): $839-841 \underline{\text { DOI] }}$

Sun J, Gale B J S, Reid D T. Coherent synthesis using carrier-envelope phase controlled pulses from a dual-color femtosecond optical parametric oscillator. Opt Lett, 2007, 32(11): 1396-1398 [DOI]

40 Sun J, Gale B J S, Reid D T. Dual-color operation of a femtosecond optical parametric oscillator exhibiting stable relative carrier-envelope phase-slip frequency. Opt Lett, 2006, 31(13): 2021-2023 [DOI]

41 Reid D T, Cormack I G, Wadsworth W J, et al. Soliton self-frequency shift effects in photonic crystal fibre. J Mod Opt, 2002, 49(5): $757-767 \underline{\mathrm{DOI}]}$

$42 \mathrm{Yu} \mathrm{T} \mathrm{J,} \mathrm{Hong} \mathrm{K} \mathrm{-H,} \mathrm{Choi} \mathrm{H} \mathrm{-G,} \mathrm{et} \mathrm{al.} \mathrm{Precise} \mathrm{and} \mathrm{long-term} \mathrm{stabilization} \mathrm{of} \mathrm{the} \mathrm{carrier-envelope} \mathrm{phase} \mathrm{of} \mathrm{femtosecond} \mathrm{laser} \mathrm{pulses}$ using an enhanced direct locking technique. Opt Express, 2007, 15(13): 8203-8211 [DOI] 